\title{
A photometric catalogue of galaxies in the cluster Abell $496^{\star, \star \star}$
}

\author{
E. Slezak ${ }^{1}$, F. Durret ${ }^{2,3}$, J. Guibert ${ }^{4}$, and C. Lobo ${ }^{5}$ \\ 1 Observatoire de la Côte d'Azur, BP. 229, F-06304 Nice Cedex 4, France \\ 2 Institut d'Astrophysique de Paris, CNRS, Université Pierre et Marie Curie, 98bis Bd. Arago, F-75014 Paris, France \\ 3 DAEC, Observatoire de Paris, Université Paris VII, CNRS (UA 173), F-92195 Meudon Cedex, France \\ ${ }^{4}$ CAI et Observatoire de Paris, 61 avenue de l'Observatoire, F-75014 Paris, France \\ 5 Osservatorio Astronomico di Brera, via Brera 28, I-20121 Milano, Italy
}

Received February 8; accepted August 26, 1999

\begin{abstract}
We present two catalogues of galaxies in the direction of the rich cluster Abell 496. The first one includes 3879 galaxies located in a region of roughly $\pm 1.3^{\circ}$ from the cluster centre. It has been obtained from a list of more than 35000 galaxy candidates detected by scanning part of a Schmidt photographic plate taken in the $b_{\mathrm{J}}$ band. Positions are very accurate in this catalogue but magnitudes are not. This led us to perform CCD imaging observations in the $V$ and $R$ bands to calibrate these photographic magnitudes. A second catalogue gives a list of galaxies with CCD magnitudes in the $V$ (239 galaxies) and $R$ (610 galaxies) bands for a much smaller region in the centre of the cluster.

These two catalogues will be combined with a redshift catalogue of 466 galaxies (Durret et al. 1999) to investigate the cluster properties at optical wavelengths (Durret et al. in preparation), as a complement to previous X-ray studies by a member of our group (Pislar 1998).
\end{abstract}

Key words: galaxies: clusters: individual: Abell 496; galaxies: clusters of

\section{Introduction}

Abell 496 is a cluster of richness class 1 (Abell 1958) located at an average redshift $z=0.0331$. We performed a detailed analysis of this cluster from the X-ray point of view, based on ROSAT PSPC data (Pislar 1998).

Send offprint requests to: E. Slezak, e-mail: slezak@obs-nice.fr

* Based on plates scanned with the MAMA microdensitometer at CAI, Paris and on observations collected at the European Southern Observatory, La Silla, Chile.

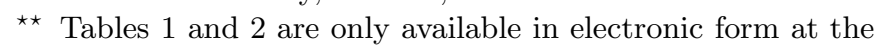
CDS via anonymous ftp to cdsarc.u-strasbg.fr (130.79.128.5) or via http://cdsweb.u-strasbg.fr/Abstract.html
In the optical, no photometric data had been published, and about 150 redshifts were available from the literature at the beginning of our study (Beers et al. 1991; Malumuth et al. 1992). We undertook a complete analysis of this cluster, with the aim of obtaining both photometric and redshift data at optical wavelengths and couple them with X-ray data. We present here our photometric data. The redshift catalogue is published in a companion paper (Durret et al. 1999) and the analysis of all these combined optical data will be presented in a coming paper (Durret et al. in preparation).

\section{The photographic plate data}

The photometric catalogue of the galaxies in the direction of the Abell 496 cluster of galaxies was obtained by processing the field number 621 in the SRC-J Schmidt atlas. Part of the glass copy of this blue plate (IIIaJ+GG385) was investigated in June 1993 with the MAMA (Machine à Mesurer pour l'Astronomie) facility located at the Centre d'Analyse des Images at the Observatoire de Paris and operated by CNRS/INSU (Institut National des Sciences de l'Univers). Due to the cluster location with respect to the plate boundaries, only a $2.5^{\circ} \times 2.5^{\circ}$ square region roughly centered on the cluster coordinates could indeed be searched for objects. At the cluster redshift, this limited scan is however large enough to secure a correct investigation of the overall cluster area (radius of $\sim 4.5 h_{50}^{-1} \mathrm{Mpc}$ at least).

The algorithms involved in the MAMA on-line mode available at that time for detecting and measuring astronomical sources are summarized in Slezak et al. (1998). Basically, objects appear to be defined as a set of connected pixels with intensity higher than typically a 2 sigma threshold above the local sky background and they are described by their flux (the sum of backgroundsubtracted pixel values, which lead to what is called 
hereafter a "plate" magnitude), their area and their elliptical shape parameters. This approach is efficient in most cases. However, one must expect that this simple vision model fails for crowded fields where the probability to get blended objects increases drastically when low detection thresholds are applied. The galactic latitude of Abell 496 is $b_{\mathrm{II}} \simeq-34.4^{\circ}$ and some blends with stars are indeed included in the 35541 individual sources listed in the on-line catalogue we were provided with (cf. the final visual check of the galaxy candidates which is described below).

The astrometric reduction of the whole catalogue was performed with respect to 98 stars of the PPM star catalogue (Roeser \& Bastian 1991) spread over the plate using a $3^{\text {rd }}$-order polynomial fitting. The residuals of the fit yielding the instrumental constants were smaller than 0.20 arcsecond and the astrometry of our catalogue indeed appears to be very good, as confirmed by crosschecking galaxy coordinates with the literature or the APM database (mean average separation: 0.7 arcsec) and by our multi-object fibre spectroscopy follow-up where the galaxies were always found to be very close to the expected positions.

The CCD observations needed to calibrate accurately the Schmidt plate were not available at that time. Hence, a preliminary photometric calibration of these photographic data has been done using galaxies with known total blue magnitude. The use of catalogued stars was rejected since such high-surface brightness objects suffer from too severe saturation effects (coming both from the emulsion itself and from the electronic settings of the MAMA facility). So, 40 galaxies available in the Lyon Extragalactic Database (LEDA, Paturel et al. 1997) were selected and their magnitudes compared to their measured blue fluxes providing that no close or overlapping objects were present as checked from a small scan around each LEDA galaxy. These 31 undisturbed objects span a 3 magnitude range, but in a very non uniform way. Hence, the $3 \sigma$ clipping routine used to compute the best fit further discarded 15 objects. The final $\mathrm{rms}$ on the zero-point is unfortunately not better than $0.4 \mathrm{mag}$ owing to: i) the quite large uncertainty quoted for the total magnitude estimate in the database along with their irregular distribution, ii) the limited magnitude range, and iii) differences in the involved flux estimates ("plate" vs. total magnitude).

A basic star-galaxy separation has been performed mainly with respect to a classical surface brightness criterion. As usual for glass copies of survey plates, the ability of this criterion to discriminate drops sharply for objects fainter than approximately $19^{\text {th }}$ magnitude. However completeness and purity of any catalogue are most of the time competing goals. So, another test based on the elongation was then performed in order to reject linear plate flaws, as well as to pick bright elongated galaxies first classified as stars due to strong saturation effects. Finally, spurious detections that occur around very bright stars (area greater

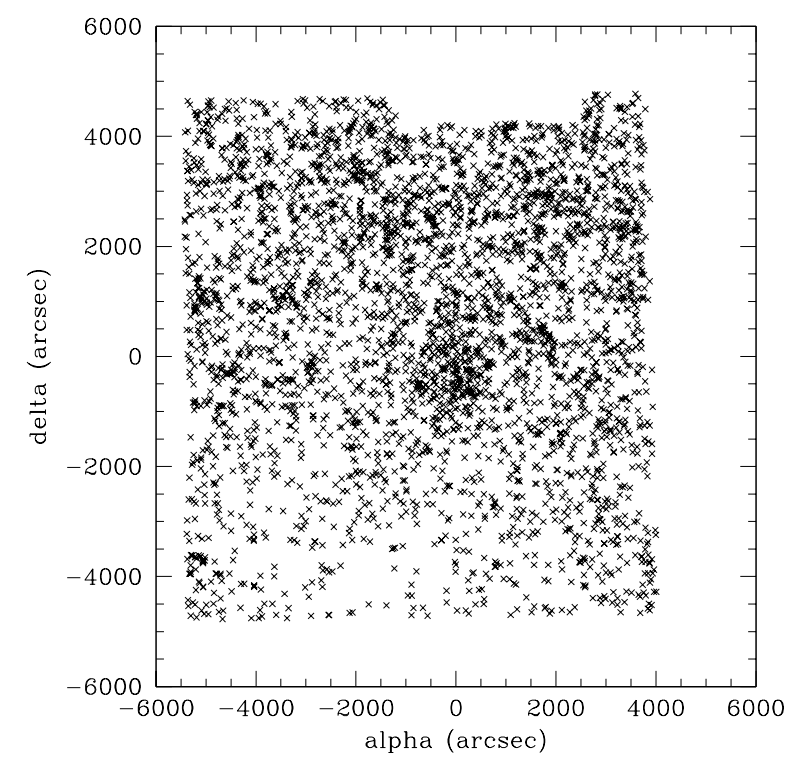

Fig. 1. Spatial distribution of 3879 galaxy candidates in the field

than $10^{3}$ pixels) due to an incorrect estimate of the local background were tentatively removed by checking their location with respect to these bright objects (the detection processing included no smoothing). Down to the detection limit, we obtained a list of more than 4000 galaxy candidates over our SRC-J 621 blue field to the detection limit.

The differential luminosity distribution of our catalogue of diffuse objects indicates that this sample is complete down to the $b_{\mathrm{J}}=19.5$ magnitude (see Fig. 2). However, its purity is less than the usual 95\% level for high galactic latitude fields (cf. Slezak et al. 1998). Our spectroscopic run indeed indicates a contamination level by stars at least three times higher $(\simeq 20 \%$, see Durret et al. 1999). Such a low success rate may partly be ascribed to the overall image quality at the plate corner where Abell 496 is located. As usual for Schmidt plates the PSF may indeed be quite poor at the borders, which randomly increases the fuzziness of otherwise point-like objects and thereby leads to parameter estimates closer to those of diffuse objects than to genuine star-like ones (cf. Fig. 1 where an overdensity is clearly visible at the edges of the plate). More generally, one can also question the efficiency of the classification procedure itself when the number density of stars is very high. In fact, a selection mostly based on a surface brightness criterion implies keeping $\simeq 5 \%$ of the total number of stars in order to select most of the galaxies. Hence, in most cases, the contamination level of a quite complete galaxy sample unavoidably increases with the star number density. The galactic latitude of Abell 496 is $b_{\mathrm{II}}=-34.4^{\circ}$, which is quite close to the Galactic plane and may explain the 


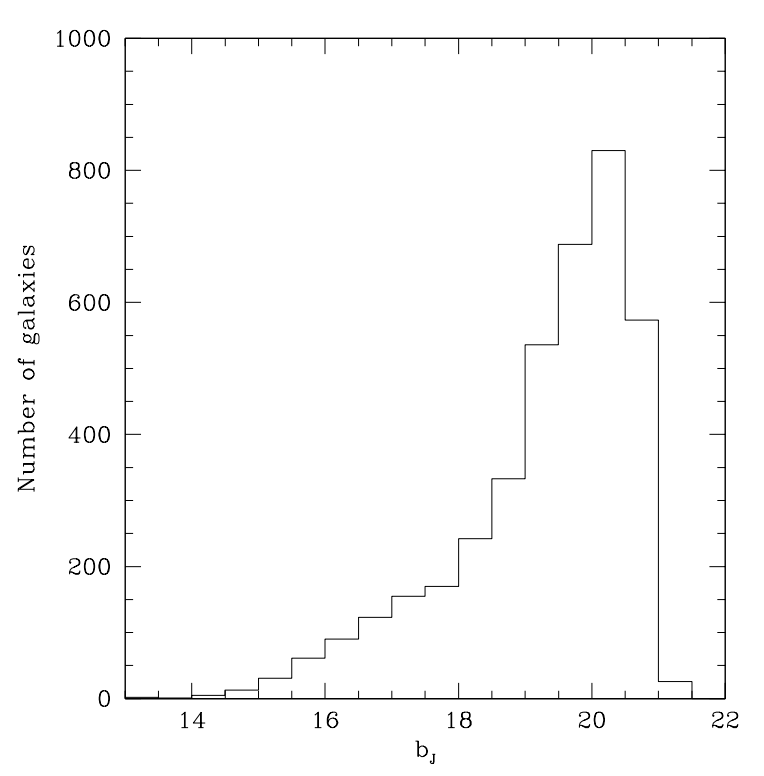

Fig. 2. Magnitude distribution in the $b_{\mathrm{J}}$ band of the 3879 galaxy candidates in Abell 496 photographic plate field

high absolute number of misclassified single stars. But, for the present data, blended stars identified as a single diffuse object is the main explanation, as first noticed during the spectroscopic run and partly expected from the involved detection software. This is confirmed by a check of our list of bright $\left(b_{\mathrm{J}}<17.5\right)$ galaxy candidates against the APM list for the \#621 SRC-J field. Among our 449 candidates within the same celestial zone, 389 objects are described as nebular in the APM catalogue, 52 are classified as star-like, 1 is related to a plate flaw while 10 have no close counterpart. As evidenced by a visual check using the DSS, among these 62 discrepant objects, there are 8 genuine galaxies, 2 asymmetrical objects, 40 blended stars, 8 single bright stars (important saturation, diffraction spikes), and 4 star-like objects. So, it appears that only 52 objects are misclassified (12\% of the total number of candidates), out of which $75 \%$ are merged images, plus two asymmetrical objects.

On one hand, this $\simeq 15 \%$ contamination level is disturbing for studies involving individual objects picked among galaxy candidates. On the other hand, its net effect for statistical studies is only a decrease of the contrast for the signal of interest providing that the misclassified stars are randomly distributed. So, for such applications, the present photometric catalogue of Abell 496 certainly remains valuable.

Table 1 lists the catalogue of galaxy candidates obtained from the SRC-J 621 plate in the $2.5^{\circ} \times 2.5^{\circ}$ field of Abell 496. Note that the 101 misclassified stars we were able to identify during our spectroscopic follow-up have been rejected, as well as the 54 objects selected by a visual check (the 52 misclassified objects and 2 asymmetrical objects described above), yielding 3879 entries. The

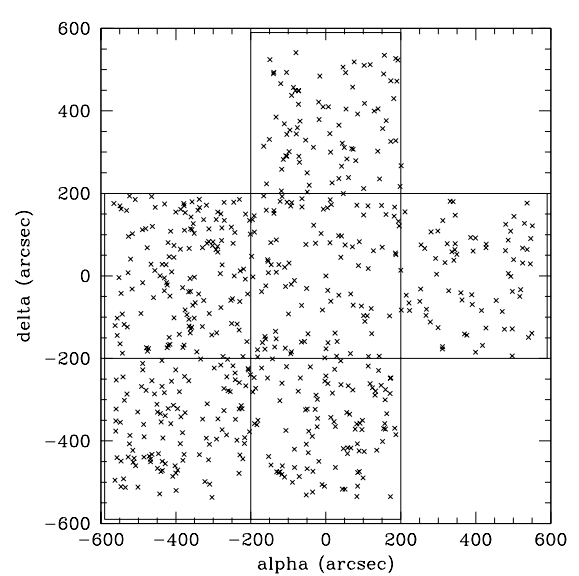

Fig. 3. Positions of the 610 galaxies in our CCD catalogue, with the observed fields superimposed. The size of each field is $6.4 \times 6.4 \operatorname{arcmin}^{2}$. Positions are drawn relatively to the centre defined in the text

meaning of the columns is the following:

(1) running number;

(2) to (4) right ascension (equinox 2000.0);

(5) to (7) declination (equinox 2000.0);

(8) half-major axis (arcseconds);

(9) excentricity $e$ defined as $\sqrt{1-\left(\frac{b}{a}\right)^{2}}$, where $a$ and $b$ are the major and minor axes respectively;

(10) position angle of the major axis (from North to East);

(11) $b_{\mathrm{J}}$ magnitude;

(12) and (13) $X$ and $Y$ positions in arcsecond relative to the centre defined as that of the diffuse X-ray emission of the cluster (see Sect. 3.1);

(14) distance to cluster center in arcseconds;

(15) MAMA catalogue reference number.

\section{The CCD data}

\subsection{Description of the observations}

The observations were performed with the Danish $1.5 \mathrm{~m}$ telescope at ESO La Silla during 2 nights on November 2 and 3,1994 . A sketch of the observed fields with the positions of the galaxies appearing in the CCD catalogue is displayed in Fig. 3. The central field was centered on the coordinates of the cluster center, defined both by the position of the $\mathrm{cD}$ and by the centroid of the X-ray emission (Pislar 1998): $04^{\mathrm{h}} 33^{\mathrm{m}} 37.9^{\mathrm{s}},-13^{\circ} 15^{\prime} 47^{\prime \prime}$ (equinox 2000.0). There was almost no overlap between the various fields (only a few arcseconds). Johnson $V$ and $R$ filters were used. Exposure times were $10 \mathrm{mn}$ for all fields; $1 \mathrm{mn}$ exposures were also taken for a number of fields with bright stars in order to avoid saturation. The detector was CCD \#28 with $1024^{2}$ pixels of $24 \mu \mathrm{m}$, giving a sampling on the sky of $0.377^{\prime \prime} /$ pixel, and a size of $6.4 \times 6.4 \mathrm{arcmin}^{2}$ for each field. The seeing was poor: $2.4^{\prime \prime}$ the first night and $1.1^{\prime \prime}$ the second night. 


\subsection{Data reduction}

Corrections for bias and flat-field were performed in the usual way with the IRAF software. Only flat fields obtained on the sky at twilight and dawn were used; dome flat fields were discarded because they showed too much structure.

Each field was reduced separately. The photometric calibration took into account a zero point correction, the airmass $(\mathrm{AM})$ and the color index $(V-R)$. For the first night, the best fits to obtain the calibrated magnitudes $V_{\text {cal }}$ and $R_{\text {cal }}$ from the measured magnitudes $V_{\mathrm{m}}$ and $R_{\mathrm{m}}$ were obtained for the following parameters:

$V_{\text {cal }}=V_{\mathrm{m}}-2.2-0.47 A M+0.025(V-R)_{\text {cal }}$,

$R_{\text {cal }}=R_{\mathrm{m}}-2.07-0.51 A M+0.0046(V-R)_{\mathrm{cal}}$, with

$(V-R)_{\text {cal }}=-0.14+0.04 A M+1.02(V-R)_{\mathrm{m}}$.

For the second night, the corresponding relations were:

$V_{\text {cal }}=V_{\mathrm{m}}-2.711-0.075 A M+0.009(V-R)_{\text {cal }}$,

$R_{\text {cal }}=R_{\mathrm{m}}-2.677-0.071 A M+0.072(V-R)_{\text {cal }}$, with

$(V-R)_{\mathrm{cal}}=-0.034-0.004 A M+0.941(V-R)_{\mathrm{m}}$.

The error bars on the various calibration parameters are the following: $V$ band, first night: $-2.2 \pm 0.1,-0.47 \pm 0.08$, $0.025 \pm 0.025 ; R$ band, first night: $-2.07 \pm 0.15,-0.51 \pm$ $0.11,0.0046 \pm 0.037 ; V$ band, second night: $-2.711 \pm 0.004$, $-0.075 \pm 0.004,0.009 \pm 0.003 ; R$ band, second night: $-2.677 \pm 0.002,-0.071 \pm 0.002,0.072 \pm 0.002$. Note that the color term for the first night in the $R$ band is in fact undefined; however, we have kept it for the sake of coherence between all calibrations. It also appears that both the tranformation and extinction terms for this first night strongly differ from those for the second night. For the first night, the range in standards and in air-mass is smaller, which may explain the discrepancy, together with perhaps poorer photometric conditions.

Since the exposure times were the same in $V$ and $R$, a number of galaxies were detected in $R$ but not in $V$. For these objects, a photometric calibration was performed without using a color term. The corresponding relations were: $R_{\text {cal }}=R_{\mathrm{m}}-2.06-0.51 A M$ for the first night and $R_{\text {cal }}=R_{\mathrm{m}}-2.70-0.03 A M$ for the second night. The corresponding error bars on these parameters are: $-2.06 \pm 0.14,-0.51 \pm 0.11,-2.70 \pm 0.04,-0.028 \pm 0.028$.

Objects were automatically detected using the DAOPHOT/ DAOFIND tasks of IRAF. This task first performs a convolution with a Gaussian with characteristics set according to the seeing in each frame (FWHM of the star-like profiles in the image) as well as the CCD readout noise and gain. Objects are then identified as the peaks of the convolved image which are higher than a given threshold above the local sky background (chosen as approximately equal to 4 times the rms of the mean sky level on the image). A list of detected objects is thus produced and interactively corrected on the displayed

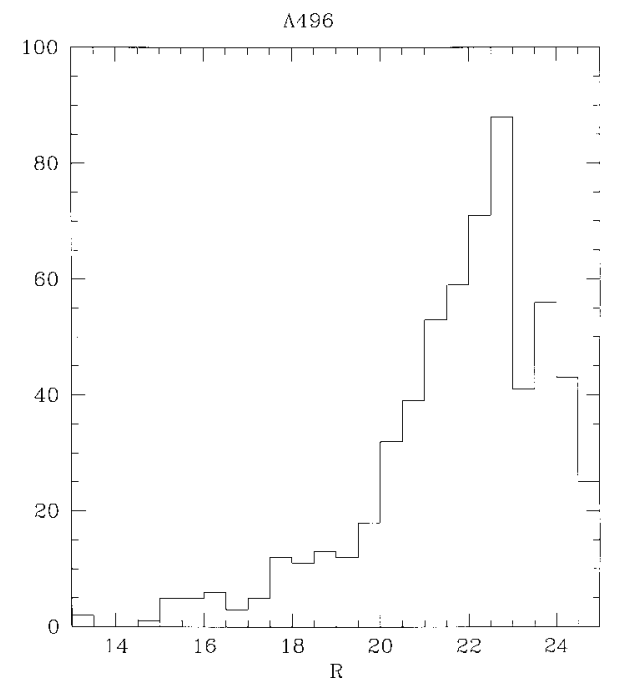

Fig. 4. Histogram of the $R$ magnitudes of the 610 galaxies in the CCD catalogue

image so as to discard spurious objects, add undetected ones (usually close to the CCD edges) by modifying the detection parameters and dispose of false detections.

We used the package developed by Le Fèvre (Le Fèvre et al. 1986) to obtain for each field a catalogue with the $(x, y)$ galaxy positions, isophotal radii, excentricities, major axis, position angles, and $V$ and $R$ magnitudes within the 26.5 isophote. To perform a star-galaxy classification based on the compactness parameter described in Le Fèvre et al. (1986), we measured the required information for each object with dedicated software we developed. Very bright stars are saturated and deviate significantly from the Gaussian-like PSF involved in the computation of the classification parameter. They are therefore classified as non stellar objects with this criterion and had to be eliminated manually. The rms accuracy on these CCD magnitudes is about 0.1 magnitude, and their errors are in all cases smaller than 0.2 magnitude.

The astrometry of this CCD catalogue is accurate to about 2.0 arcsec as verified from the average mutual angular distance between CCD and MAMA equatorial coordinates for the galaxies included in both catalogues.

The histogram of the $R$ magnitudes in the CCD catalogue is displayed in Fig. 4. The turnover value of this histogram is located around $R \simeq 22.5-23$, suggesting that our catalogue is complete up to $R \sim 22$.

The histogram of the $(V-R)$ colour is plotted in Fig. 5 .

\subsection{Transformation laws between the photometric systems}

By identifying galaxies in our CCD catalogue with objects in our photographic plate catalogue, we derived the following calibration relations between our photographic plate $b_{\mathrm{J}}$ magnitudes and our $R$ CCD magnitudes: first night: 


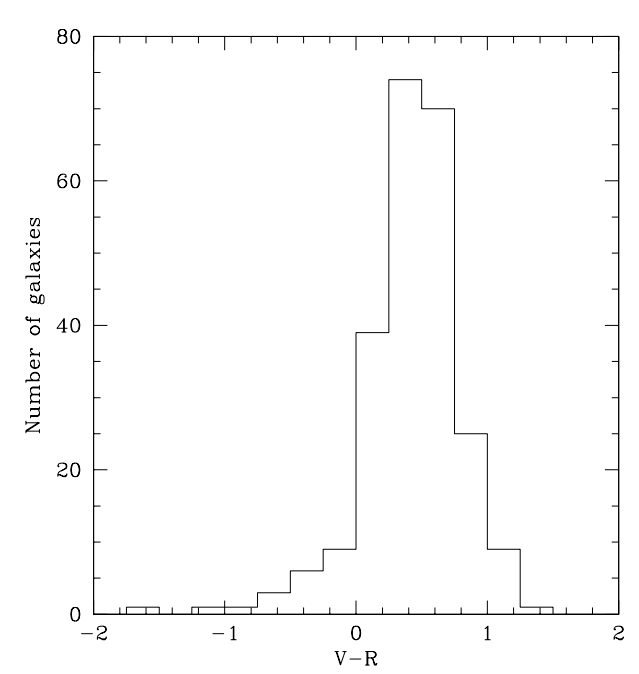

Fig. 5. Distribution of the $(V-R)$ colour as a function of $R$ for the 239 galaxies detected in the $V$ band in our CCD catalogue

$R_{\mathrm{CCD}}=b_{\mathrm{J}}-0.28, \mathrm{rms}=0.05$ (5 galaxies); second night: $R_{\mathrm{CCD}}=b_{\mathrm{J}}-0.30, \mathrm{rms}=0.01$ (9 galaxies); mean value: $R_{\mathrm{CCD}}=b_{\mathrm{J}}-0.28 \pm 0.01, \mathrm{rms}=0.01$ (10 galaxies $)$. We did not include any colour term, because it did not make the fit any better. The difference between the observed $R$ band CCD magnitude $R_{\mathrm{CCD}}$ and the $R$ magnitude calculated from $b_{\mathrm{J}}$ with the above formula is plotted in Fig. 6 as a function of the CCD $R$ magnitude. This difference is small and does not appear to increase with magnitude.

\subsection{The CCD catalogue}

The CCD photometric data for the galaxies in the field of Abell 496 are given in Table 2. The meaning of the columns is the following:

(1) Running number;

(2) to (4) right ascension (equinox 2000.0);

(5) to (7) declination (equinox 2000.0);

(8) 26.5 magnitude isophotal radius in arcseconds;

(9) excentricity $e$ defined as $\sqrt{1-\left(\frac{b}{a}\right)^{2}}$, where $a$ and $b$ are the major and minor axes respectively;

(10) position angle of the major axis (in degrees from North to East);

(11) and (12) $V$ and $R$ magnitudes;

(13) and (14) $X$ and $Y$ positions in arcseconds relative to the X-ray centre.

\section{Summary}

Our redshift catalogue is submitted jointly in a companion paper (Durret et al. 1999). Together with the catalogues presented here, it will be used to give an interpretation of the optical properties of Abell 496 (Durret et al. in

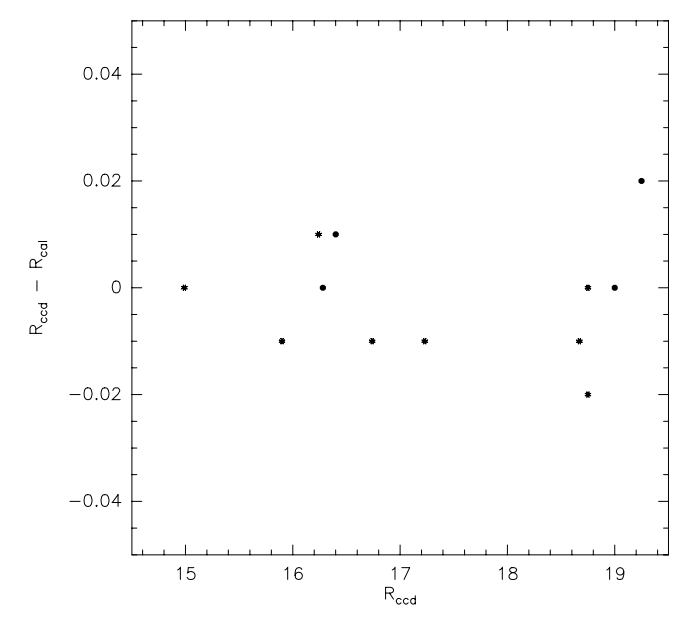

Fig. 6. Difference between the $R$ band CCD magnitude and the $R$ band magnitude calculated from the photographic $b_{\mathrm{J}}$ magnitude (see text) as a function of observed $R$ band CCD magnitude

preparation), in relation with the X-ray properties of this cluster (Pislar 1998).

Acknowledgements. We are very grateful to the MAMA team at Observatoire de Paris for help when scanning the photographic plate, and to Cláudia Mendes de Oliveira for her cheerful assistance at the telescope. The help of Françoise Warin in the data reduction is gratefully acknowledged. We thank the referee, John Huchra, for many comments which allowed us to improve the paper and in particular for pointing out mistakes which led us to redo the photometrical calibration. C.L. acknowledges financial support by the CNAA (Italia) fellowship reference D.D. No. 37 08/10/1997.

\section{References}

Abell G.O., 1958, ApJS 3, 211

Beers T.C., Forman W., Huchra J.P., Jones C., Gebhardt K., 1991, AJ 102, 1581

Durret F., Felenbok P., Lobo C., Slezak E., 1999, A\&AS (in press)

Le Fèvre O., Bijaoui A., Mathez G., Picat J.P., Lelièvre G., 1986, A\&A 154, 92

Malumuth E.M., Kriss G.A., Van Dyke Dixon W., Ferguson H.C., Ritchie C., 1992, AJ 104, 495

Paturel G., Andernach H., Bottinelli L., Di Nella H., Durand N., et al., 1997, A\&AS 124, 109

Pislar V., 1998, Ph.D. Thesis, Université Paris 6

Roeser S., Bastian U., 1991, PPM Star Catalogue, Spektrum Akademischer Verlag. Heidelberg, Berlin, New-York

Slezak E., Durret F., Guibert J., Lobo C., 1998, A\&AS 128, 67 